\title{
PHENIX detector upgrades for enhanced physics programs
}

\author{
Yuji GOTO*† \\ (RIKEN Nishina Center for Accelerator-Based Science and RIKEN BNL Research Center) \\ E-mail: goto@bnl.gov
}

The PHENIX collaboration proposes detector upgrades for enhanced physics programs using full luminosity of recent upgraded RHIC facility. A major upgrade of the midrapidity spectrometer consists of a solenoidal magnet for high-resolution tracking surrounded by electromagnetic and hadronic calorimeters with uniform coverage over $|\eta|<1$. The upgrade enables a crucial jet physics program to investigate the nature of the strongly-coupled quark-gluon plasma. Additional tracking layers and a preshower will expand the program to include heavy-flavor, neutral pion, low-mass dilepton, and direct photon measurements. Moreover we propose a forward-rapidity spectrometer upgrades with the open geometry. It has been designed for the study of cold nuclear matter effects in proton- and deuteron-nucleus collisions, precision measurements of single transverse-spin asymmetries for the Drell-Yan process, and measurements of novel observables in jet production in transversely polarized proton collisions.

XXI International Workshop on Deep-Inelastic Scattering and Related Subjects 22-26 April, 2013

Marseilles, France

\footnotetext{
*Speaker.

$\dagger$ On behalf of the PHENIX collaboration.
} 


\section{Introduction}

The PHENIX experiment proposes substantial detector upgrades for long-term enhancement for major physics programs using upgraded RHIC accelerator [1]. We want to understand the nature of the strongly-coupled quark-gluon plasma (sQGP) discovered experimentally at RHIC. In order to understand the sQGP, it is essential to know its initial state, or the nature of quarks and gluons in the nucleus. We also want to understand the spin structure of the nucleon using polarized proton collisions at RHIC. For understanding quark-gluon structure of the nucleus and the spin-structure of the nucleon, we aim at building three-dimensional picture of the nucleus and the nucleon.

The first stage of the upgrades to the PHENIX detector is a construction of a compact jet detector at midrapidity with high-rate capability. Precision jet, dijet, and photon-jet measurement is a major topic to understand the nature of the sQGP. There are options for upgrades which extend midrapidity tracking and preshower detectors. They will enable us heavy-flavor quarkonia and internal-jet structure measurements in addition.

The open geometry of the forward direction of the midrapidity detector upgrades allow for the addition of a forward spectrometer. The forward detector upgrades give wide kinematic coverage of photon, jet, leptons, and identified hadrons which serves for understanding transverse-momentum dependent (TMD) quark-gluon structure of the nucleon and the nucleus, and measurement of the nuclear gluon distribution and search for gluon saturation at small- $x$. We expect the upgraded detector to be applied also in the electron-ion collisions in the future at RHIC (or eRHIC), where another picture of quark-gluon structure of the nucleon and the nucleus is given, and precision understanding of sQGP is also given by knowing the initial state of the relativistic heavy-ion collisions. The upgrades towards a detector at eRHIC is covered by another article in these proceedings [2].

\section{Midrapidity upgrades}

We propose to construct a compact jet detector for the midrapidity upgrades on the existing infrastructure in the PHENIX interaction region. It has full-azimuthal coverage in a fiducial region of $|\eta|<1$. We apply a solenoid for high-resolution tracking in a small volume, and implement electromagnetic and hadronic calorimetry with data acquisition capable of recording more than $10 \mathrm{kHz}$. The detector performance requires to distinguish jets from background fakes with use of recent upgraded RHIC luminosity. The magnetic solenoid has $2 \mathrm{~T}$ magnetic field, $70 \mathrm{~cm}$ inner radius and less than $1 X_{0}$ radiation length. The electromagnetic calorimeter is a compact calorimeter with accordion structure of tungsten and scintillating fiber. It is required to have a high degree of segmentation $(0.03 \times 0.03$ in pseudorapidity $\eta$ and azimuth $\phi)$ and an energy resolution $\sigma_{E} / E$ of about $15 \% / \sqrt{E}$. It is readout with Silicon photomultiplier (SiPM) which is small, has high gain, requires no high voltage, and works in a magnetic field. The hadronic calorimeter is a steelscintillator calorimeter which has about 5 interaction length. Wavelength shifting fiber is embedded in scintillator for light collection. It is required to have a granularity of $0.1 \times 0.1$ in $\eta$ and $\phi$ and $\sigma_{E} / E$ of about $100 \% / \sqrt{E}$. It works also for flux return of the magnetic field from the solenoid.

In the existing PHENIX detector, the jet-probe measurements have been performed for nuclear modification factor measurement [3] and azimuthal anisotropy of hadron production with respect to the reaction plane [4]. The nuclear modification factor shows suppression of single hadron yields 
compared to expectations from $p+p$ collisions. It indicates jet quenching, significant loss of energy for partons traversing the QGP. The azimuthal anisotropy shows weakly-coupled radiative energy loss model cannot explain the measurement. New data from LHC showed measurements of fully reconstructed jets [5, 6] which showed energy loss for jets and unmodified $\Delta \phi$ distribution which means no broadening in traversing the matter. The precision measurements of jet, dijet, and photonjet have given us significant extension of kinematic range and new information of jet modification to understand the nature of the sQGP. It is important to have fully-reconstructed jet measurements at RHIC to study temperature dependence ( $>300 \mathrm{MeV}$ at RHIC [7] and $>420 \mathrm{MeV}$ at LHC [8]), and have more flexible study at RHIC with different energies and nuclear species.

There are options for midrapidity upgrades which extend charged-particle tracking for high resolution momentum measurement with additional silicon layers, and preshower detector with fine segmentation. Improved tracking and improved electron identification will enable us good separation of Upsilon states. Improved separation of single photon with preshower detector will also improved reach of photon $/ \pi^{0}$ separation up to $50 \mathrm{GeV}$ instead of separation up to $10 \mathrm{GeV}$ with only the electromagnetic calorimeter. These additions will expand the program to include heavy-flavor, neutral pion, low-mass dilepton, and direct photon measurements. Figure 1 shows an engineering drawing of the upgraded detector.

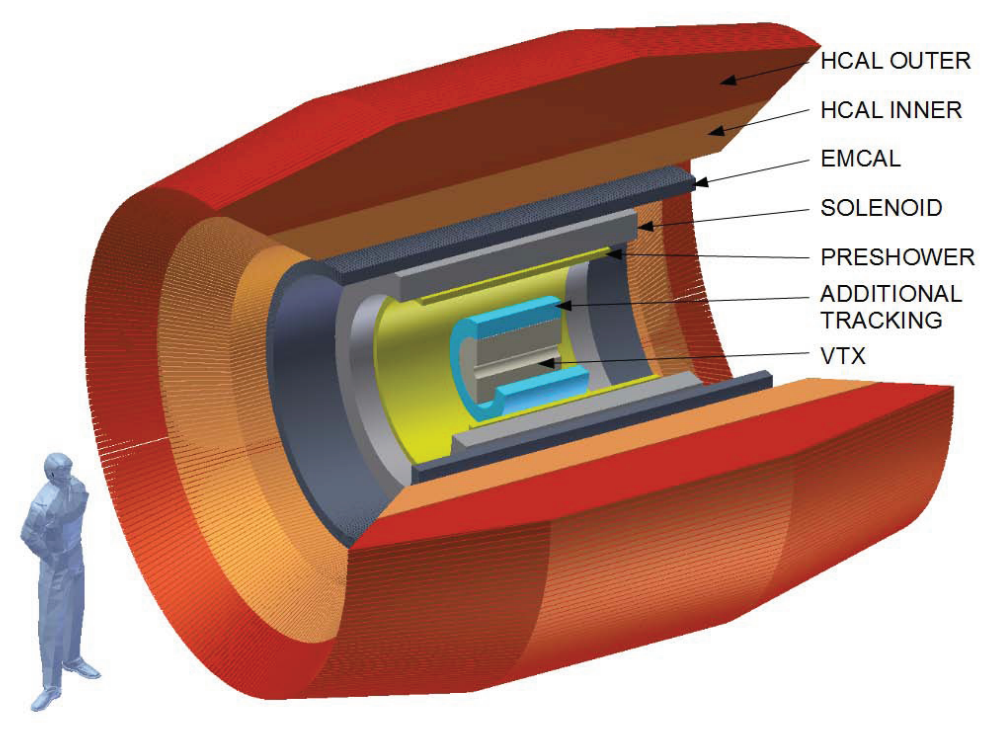

Figure 1: An engineering drawing of the midrapidity upgrades showing the inner silicon tracker (VTX), the solenoid, the calorimeters, and additional charged-particle tracking and preshower detectors.

\section{Forward upgrades}

The proposed midrapidity upgrades replaces the present magnet with a solenoid, and removes the large iron yoke at forward rapidity that provides the hadron absorber for the muon detectors. The open geometry will allow for the addition of a forward spectrometer covering forward rapidity 
region, $1<\eta<4$ with capability of measuring hadrons, photons, electrons, muons and jets. We have been investigating requirements for detector design and performance of the forward upgrades consisting of charged-particle tracking (e.g. GEM), particle identification (e.g. RICH), electromagnetic and hadronic calorimeters.

One of the physics topics with the forward upgrades is Cold Nuclear Matter (CNM) effects in proton- and deuteron-nucleus collisions. We want to measure nuclear gluon distribution, $G_{A}(x)$, to know initial state of heavy-ion collisions and understand the sQGP. It is important to search gluon suppression, or suppression of $G_{A}(x)$, at small- $x$ and verify the Color Glass Condensation (CGC) framework which is an effective field theory to describe the saturated gluon [9. We also want to know the pQCD mechanism of the energy loss of partons in CNM, its relation to transverse momentum broadening, and detailed hadronization and time scales.

In the present PHENIX detector, the CNM effects are measured with $J / \psi$ and hadron-hadron correlations over a broad range of rapidity [10]. It is sensitive to extended range of $x$. Open heavyflavor and a first look at Drell-Yan will be performed with forward vertex detector (FVTX) which was installed in 2012, and compared with $J / \psi$ data. Prompt photon will also be performed in the muon piston region with present forward electromagnetic calorimeter (MPC) [11] and preshower detector (MPC-EX) [12] which will be installed in 2014. The forward upgrades will add measurements of quarkonia, vertex-tagged open heavy-flavor, inclusive hadrons and fully- reconstructed jets, jet-jet correlation, and Drell-Yan with much more extended kinematic reach, smaller statistical and systematic uncertainties and different collision energies and nuclear species.

Another physics topic is measurements of single transverse-spin asymmetry. The asymmetries have been measured in the Fermilab fixed-target experiment with transversely-polarized proton beams, and in the RHIC transversely-polarized proton collider experiments at much higher energies as well. In order to explain the asymmetries, perturbative-QCD models have been developed. At small transverse momenta, the asymmetries have been explained with the transverse-momentum dependent (TMD) factorization framework. They have been explained with correlations between the transverse spin of the target proton and intrinsic transverse momentum of quarks in the initial state which is called Sivers mechanism [13], and described by the Sivers function. They have also been explained with correlations between quark spin and the transverse momentum of hadrons in the final state which is called Collins mechanism [14], and described by the Collins fragmentation function. At larger transverse momenta, the higher-twist effect has explained the asymmetries with spin-dependent transverse momentum components generated through quark-gluon and multi-gluon correlations with the collinear factorization framework. It has been demonstrated that the Sivers mechanism and the higher-twist effect give identical results in the region of intermediate transverse momenta [15].

The Sivers function contributes with opposite sign to the transverse- spin asymmetries in the semi-inclusive DIS process and the Drell-Yan process due to non-universality of the TMD factorization framework. This is a fundamental QCD prediction based on gauge invariance and its verification constitutes an important milestone for the field of hadron physics. It allows one to test non-perturbative aspects of QCD and the concept of factorization. The forward upgrades will enable us to measure Sivers function in the Drell-Yan process. It is a competitive program in fixedtarget experiments and in collider experiments. A comparison of the asymmetries at fixed-target energies and collider energies also provides a unique test of TMD evolution. Recently there has 
been theoretical progress in the derivation of the evolution equations for TMD parton distribution and fragmentation functions [16 17. The knowledge of the TMD evolution equations will make it possible to carry out a QCD analysis of TMD observables.

The Collins mechanism will be investigated through an azimuthal anisotropy in the distribution of hadrons in final-state jets with the forward upgrade detectors. The asymmetry of single identified hadrons described by the Collins fragmentation function and that of identified hadron pairs described by the interference fragmentation function (IFF) will give a measurement of quark transversity distribution at large $x$ which will determine the tensor charge of the nucleon and test Lattice QCD predictions for the tensor charge [18]. A comparison of quark transversity extracted from TMD Collins observables and from collinear IFF asymmetries will provide a test of the TMD framework and evolution.

Before the forward upgrades, the MPC-EX detector will enable us prompt photon asymmetry measurement which will distinguish Sivers effect and higher-twist effect, and Collins asymmetry measurement with $\pi^{0}$ correlations with jet-like clusters. There is a new possibility in collisions of polarized protons and nuclei. Transverse single-spin asymmetries in $p \uparrow+A$ collisions may have a sensitivity to the saturation scale in the nucleus. This is one of the most interesting recent development as a link between physics of CNM and spin structure of the nucleon.

\section{References}

[1] C. Aidala, N. N. Ajitanand, Y. Akiba, Y. Akiba, R. Akimoto, J. Alexander, K. Aoki and N. Apadula et al., arXiv:1207.6378 [nucl-ex].

[2] K. Dehmelt, these proceedings.

[3] A. Adare et al. [PHENIX Collaboration], Phys. Rev. C 77 (2008) 064907.

[4] A. Adare et al. [PHENIX Collaboration], Phys. Rev. Lett. 105 (2010) 142301.

[5] G. Aad et al. [ATLAS Collaboration], Phys. Rev. Lett. 105 (2010) 252303.

[6] S. Chatrchyan et al. [CMS Collaboration], Phys. Rev. C 84 (2011) 024906.

[7] A. Adare et al. [PHENIX Collaboration], Phys. Rev. Lett. 104 (2010) 132301.

[8] M. Luzum and P. Romatschke, Phys. Rev. Lett. 103 (2009) 262302.

[9] F. Gelis, E. Iancu, J. Jalilian-Marian and R. Venugopalan, Ann. Rev. Nucl. Part. Sci. 60 (2010) 463.

[10] A. Adare et al. [PHENIX Collaboration], Phys. Rev. C 77 (2008) 024912 [Erratum-ibid. C 79 (2009) 059901]: Phys. Rev. Lett. 107 (2011) 172301: arXiv:1204.0777 [nucl-ex].

[11] M. Chiu [PHENIX Collaboration], AIP Conf. Proc. 842 (2006) 1091.

[12] S. Campbell et al. [PHENIX Collaboration], arXiv:1301.1096 [nucl-ex].

[13] D. W. Sivers, Phys. Rev. D 41 (1990) 83.

[14] J. C. Collins, Nucl. Phys. B 396 (1993) 161.

[15] X. Ji, J. -W. Qiu, W. Vogelsang and F. Yuan, Phys. Rev. Lett. 97 (2006) 082002.

[16] Z. -B. Kang and J. -W. Qiu, Phys. Lett. B 713 (2012) 273.

[17] S. M. Aybat, A. Prokudin and T. C. Rogers, Phys. Rev. Lett. 108 (2012) 242003.

[18] B. U. Musch, P. Hagler, J. W. Negele and A. Schafer, Phys. Rev. D 83 (2011) 094507. 